\title{
One-dimensional metal chains on Pt vicinal surfaces
}

\author{
P. Gambardella,* M. Blanc, H. Brune, K. Kuhnke, and K. Kern \\ Institut de Physique Expérimentale, Ecole Polytechnique Fédérale de Lausanne, CH-1015 Lausanne, Switzerland
}

(Received 23 July 1999)

\begin{abstract}
High-density arrays $\left(5 \times 10^{6} \mathrm{~cm}^{-1}\right)$ of parallel nanowires have been grown using the vicinal $\mathrm{Pt}(997)$ surface as a template. Single monatomic rows of $\mathrm{Ag}$ and $\mathrm{Cu}$ can be deposited with subrow precision. We demonstrate real-time monitoring and characterization of the growth of the atomic chains as a function of temperature by thermal energy helium atom scattering. Scanning tunneling microscopy provides further insight into the structure of the metal rows. Growth mode and alloying with the Pt substrate are discussed as a function of temperature. Our results provide the basis for the creation of surfaces with a uniform distribution of wires having the same average width for the investigation of the electronic, magnetic, and chemical properties of one-dimensional and quasi-one-dimensional metal structures.
\end{abstract}

\section{INTRODUCTION}

The interest in creating well-defined structures on the nanometer scale is motivated by a multitude of research activities in the field of low-dimensional systems as well as evolving technological applications. Confinement of electrons in less than three dimensions in metal systems results in quite dramatic changes of the electronic, ${ }^{1-6}$ transport, $^{7}$ and magnetic properties ${ }^{8-13}$ of different materials. As such properties depend on the size and shape of the system under investigation, sophisticated techniques have been developed to grow tailored structures. Among these, self-organization ${ }^{14-16}$ offers the opportunity to create nanoscale patterns with densities as high as $10^{11-13} \mathrm{~cm}^{-2}$ in a fast parallel process. High densities are mandatory for the employment of integral probes to characterize the physical properties of nanoscale systems. The trade-off with respect to other patterning techniques such as e-beam or scanning probe writing is, of course, a limited choice of the available patterns and a finite width of the size distribution of the self-organized structures.

Among self-organization techniques, step decoration on periodically stepped substrates can be used to grow arrays of nanowires. Experimental work on nucleation at step edges was originally used as a method to obtain electron microscopy images of monatomic steps on rocksalt surfaces. The early studies by Bassett and Bethge ${ }^{17,18}$ showed that step ledges act as preferential nucleation sites for metal adatoms due to the increased coordination with respect to the terrace sites. However, only with the rapid advancement of thin film deposition techniques in the last two decades the role of steps in homo- and heteroepitaxial growth has been extensively characterized. Initially, molecular-beam epitaxy on vicinal surfaces has been investigated to improve layer-bylayer growth on AlAs-GaAs interfaces, ${ }^{19}$ but it was soon recognized that ordered stepped substrates can be employed as nanoscale templates for the growth of superlattices of quantum wires. ${ }^{19-23}$ Studies of metal systems have shown that growth on stepped surfaces proceeds either as a smooth step-wetting process ${ }^{3,24-27}$ or as nucleation of twodimensional (2D) islands at step edges, ${ }^{28,29}$ provided that the mean free path of the adatoms is larger than the terrace width of the substrate.

In the present study we employed $\mathrm{Pt}(997)$ as a nanotemplate to grow $1 \mathrm{D}$ wires of $\mathrm{Ag}$ and $\mathrm{Cu}$. $\mathrm{Pt}(997)$ is cut $6.5^{\circ}$ off normal with respect to the (111) crystal plane, resulting in a surface with a periodic lattice of monatomic steps that are on average $20.1 \AA$ apart. Repulsive interactions between adjacent steps suppress step meandering ${ }^{30}$ and the terrace width has a narrow gaussian distribution with standard deviation $\sigma=2.9 \AA$ (Ref. 31) (see Fig. 1). The aim of this work is twofold. First, we show that by thermal energy atom scattering (TEAS) we can achieve real-time control over monatomic wire deposition. The small spacing between the $\mathrm{Pt}$ steps and the highly periodic pattern of the substrate allow us to grow arrays of parallel nanowires of unprecedented spatial density and uniformity. Second, we want to gain detailed information on the growth processes that lead to the wire pattern formation. A more comprehensive understanding of nucleation and growth on stepped substrates is needed both on a macroscopic and a microscopic scale for the preparation of samples with well-characterized periodic wire structures down to the monatomic limit. The combination of two complementary experimental techniques such as TEAS and scanning tunneling microscopy (STM) is a powerful means to achieve this goal. TEAS offers non-invasive in situ macroscopic-scale monitoring of the wire growth at different temperatures, while STM gives access to atomic details that elude atom scattering probes. As a general trend we find that wire formation is limited at low temperature by slow edge-diffusion processes and at high temperature by heterostep-crossing and eventually by alloying between the metal adspecies and the substrate. We determine the best deposition parameters that lead to the formation of smooth $\mathrm{Ag}$ and $\mathrm{Cu}$ wires.

This paper is organized as follows: experimental details are given in Sec. II; Sec. III A and Sec. III B present the information that can be gained by TEAS and STM measurements, respectively; Ag wire growth is discussed in Secs. III C, III D, and III E, while $\mathrm{Cu}$ wire growth is reported in Sec. III F.

\section{EXPERIMENT}

TEAS experiments have been carried out in a triple-axis He spectrometer ${ }^{32,33}$ that allows an independent variation of the incidence and reflection angle between $30^{\circ}$ and $90^{\circ}$ with 


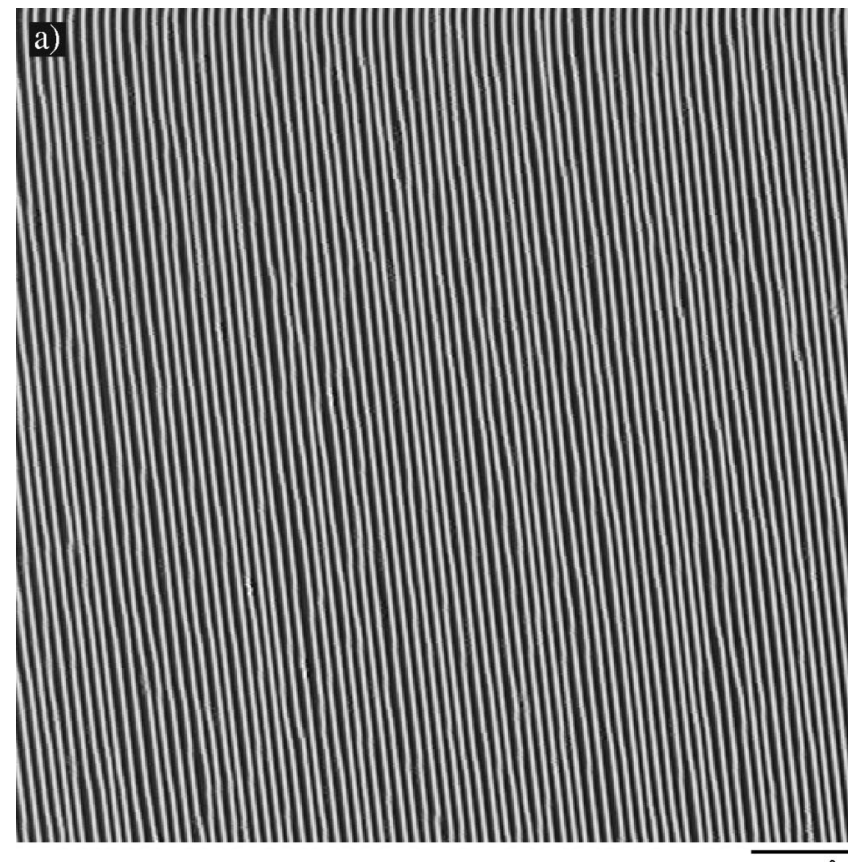

$200 \AA$

b)
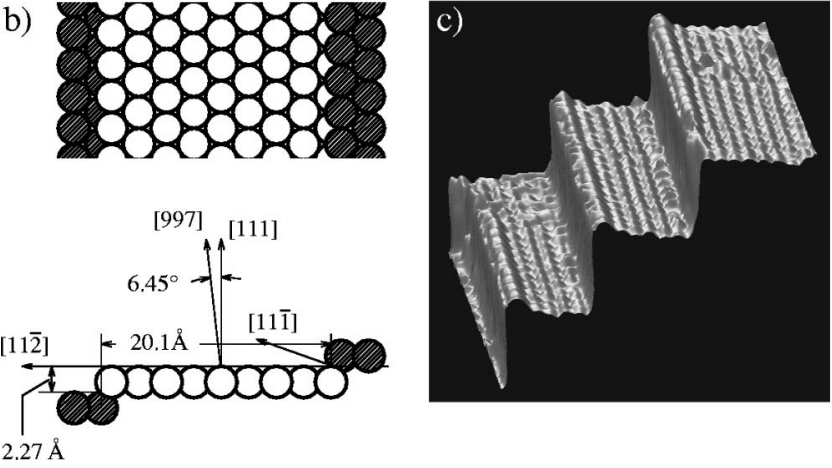

FIG. 1. (a) STM $\partial z / \partial x$ image of the clean $\operatorname{Pt}(997)$ surface. The average terrace width is $20.1 \pm 2.9 \AA$, step down direction is from right to left. Tunneling current $I=1.0 \mathrm{nA}$, sample bias $V=0.6 \mathrm{~V}$; (b) schematic of the $\operatorname{Pt}(997)$ terrace atomic structure; (c) close up of Pt steps (the $z$ scale has been exaggerated for better 3D rendering), $I=2.7 \mathrm{nA}, V=10 \mathrm{mV}$.

respect to the surface normal. The He beam spot on the sample is about $5 \mathrm{~mm}^{2}$, thereby probing the surface on a macroscopic scale. The Pt(997) surface has been prepared in situ in the scattering chamber by repeated cycles of $800 \mathrm{eV}$ $\mathrm{Ar}^{+}$sputtering at $750 \mathrm{~K}$ and annealing to $850 \mathrm{~K}$, followed by a brief exposure to $1 \times 10^{-7}$ mbar oxygen and by a flash to $T>1000 \mathrm{~K}$ to remove residual contaminant. Care has to be taken in cooling the sample at a slow rate $(<40 \mathrm{~K} / \mathrm{min})$ until $500 \mathrm{~K}$ in order to allow equilibration of the step morphology. The surface cleanliness has been checked by Auger and $\mathrm{He}$ reflectivity measurements; the base pressure in the scattering chamber was $1 \times 10^{-10}$ mbar. Even small quantities of impurities during the annealing stage result in step pinning and faceting and have to be avoided. Atoms are evaporated on the surface by e-beam heating of a crucible surrounded by a water-cooled shield, ensuring that the pressure in the chamber never raises to more than 3 $\times 10^{-10}$ mbar during evaporation. The deposition is monitored in situ with He reflectivity measurements during the evaporation.
STM images have been acquired in a different experimental setup, a home-built STM (Ref. 34), which consists of a variable temperature preparation stage and a low-temperature microscope operated at $77 \mathrm{~K}$ during this work. Sputtering and evaporation procedures were identical as the ones described in the He scattering experiments. After metal deposition in the preparation stage the sample is rapidly cooled before being transferred isothermally to the STM. The transfer process itself lasts only a few seconds and is done by a precooled wobblestick. The coverages between the He and STM samples have been cross calibrated by relative Auger peak intensities obtained using two identical spectrometers.

\section{RESULTS AND DISCUSSION}

\section{A. He scattering probing sub-monolayer growth}

On flat crystalline surfaces probing the growth of epitaxial layers by TEAS essentially consists in monitoring the intensity variations of the reflected He beam during deposition; if the scattering geometry is chosen such that the He atoms reflected from adjacent terraces interfere constructively (the so called in-phase condition), maxima in the deposition curves correspond to minima in the density of defects (diffuse scatterers) on the surface topmost layer. ${ }^{35}$ Typically, in the case of 2D layer-by-layer growth the He intensity oscillates with a period of one monolayer coverage, while for $3 \mathrm{D}$ growth it decreases monotonically to zero. On vicinal surfaces, the periodic arrangement of terraces acts as an echelette grating for He matter waves giving rise to a diffraction pattern whose most intense orders are tilted away from the specular direction. ${ }^{32}$ However, for a few diffraction orders it is still possible to obtain in-phase scattering conditions. ${ }^{36}$ We can thus monitor the average terrace defect density on our stepped surface as if we were considering scattering from a flat surface. We used in-phase scattering conditions to calibrate the $\mathrm{Ag}$ and $\mathrm{Cu}$ deposition rates. Moreover, as we discussed in a recent paper, ${ }^{36}$ on vicinal surfaces the sensitivity to different surface sites shows substantial variations depending on the scattering geometry. Grazing incidence conditions (large $\theta_{i}$ values) greatly enhance the step sensitivity, while scattering angles closer to the surface normal probe the ordering of the entire surface. By changing the scattering geometry we can select the regions of the surface we are looking at; this turns out to be particularly useful to characterize step decoration.

In what follows, we discuss some general features of the He reflectivity spectra in the particular case of $\mathrm{Ag} / \mathrm{Pt}(997)$; similar arguments hold for $\mathrm{Cu}$ as well. Figure 2 shows two deposition curves for $\mathrm{Ag}$ on $\mathrm{Pt}(997)$ at $\theta_{i}=46.9^{\circ}$ (a) and $\theta_{i}$ $=85.0^{\circ}$ (b). The intensity in (a) oscillates with a period of one monolayer coverage, demonstrating in this case a layerby-layer growth mode and serving as a precise calibration for the deposition rate. In (b) we observe a first peak at $0.13 \mathrm{ML}$ and a pronounced shoulder at $0.25 \mathrm{ML}$. Since the He reflectivity in grazing conditions depends on the defect density at the step edges and because $0.13 \mathrm{ML}$ is the nominal coverage of a monatomic wire on $\mathrm{Pt}(997)$, we attribute these peaks to the formation of the first and second Ag row along a Pt step edge, ${ }^{36}$ respectively.

The situation for $\theta_{\mathrm{Ag}} \geqslant 0.25 \mathrm{ML}$ is more complex. We do not observe further row peaks in the grazing incidence 


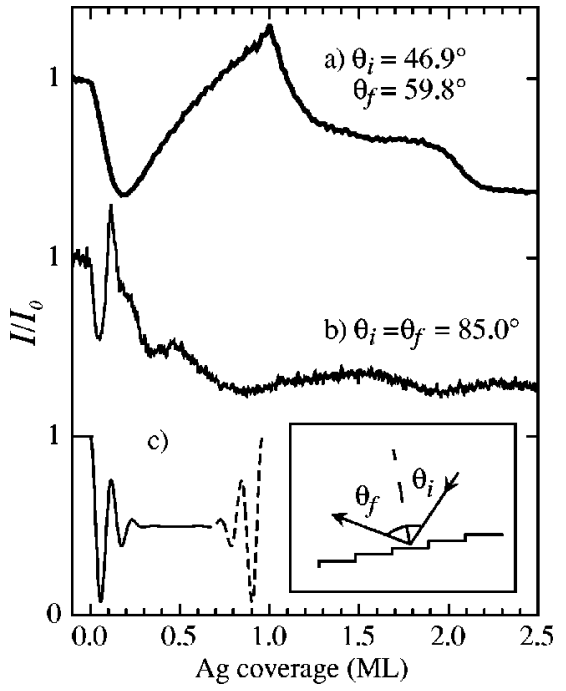

FIG. 2. Normalized intensity of the reflected He beam during deposition of $\mathrm{Ag}$ at $T=300 \mathrm{~K}$ for different scattering geometries. The contribution of the flat terraces to the reflected He intensity diminishes with increasing scattering angle while the step contribution increases. (a) Small total scattering angle: the first maximum corresponds to the completion of the first Ag monolayer and is used as an absolute coverage calibration. The completion of the second $\mathrm{Ag}$ monolayer is also observed. (b) Large scattering angle (grazing conditions): the first peak and the shoulder correspond to the formation of the first and second $\mathrm{Ag}$ atomic rows along the Pt step edges, respectively. The deposition rate is $F=4 \times 10^{-3} \mathrm{ML} / \mathrm{s}$ for both curves. The He-beam wavelength $\lambda_{\mathrm{He}}$ is $1.01 \AA$. (c) Simulated He reflectivity in grazing incidence conditions showing the effect of the row growth desynchronization for a terrace width distribution with standard deviation $\sigma=3.6 \AA$ which is close to the STM determined value of $2.9 \AA$. The dashed line indicates resynchronization, not observed on $\operatorname{Pt}(997)$ [see text].

curve; however, this does not rule out the persistence of rowby-row growth. The averaging out of the He reflected intensity is due to desynchronization of the row growth on terraces that have different widths. Since for $T \leqslant 400 \mathrm{~K} \mathrm{Ag}$ adatoms are confined to their impact terrace (see inset in Fig. 3 and Sec. III C), the coverage on each terrace is proportional to the terrace width. Thus, the rows on larger terraces are completed before those growing on smaller terraces. The same behavior has been observed by Petrovyhk et al. in the case of $\mathrm{Cu} / \mathrm{Mo}(110)$ for $T<600 \mathrm{~K} .{ }^{27}$ The simulation in Fig. 2(c) qualitatively illustrates this effect by assuming no interlayer mass transport, perfect row-by-row growth and weighting the intensities coming from the steps by the statistical occurrence of their adjacent terraces of different sizes. As the adatom coverage approaches $1 \mathrm{ML}$, "resynchronization," as shown by the dotted line in Fig. 2(c), should occur. This is not the case for the $\mathrm{Ag} / \mathrm{Pt}(997)$ system because the step edges of the growing monolayer are no longer smooth for $\theta_{\mathrm{Ag}}>0.6-0.7 \mathrm{ML}$ (see Fig. 10). In contrast, resynchronization is observed on $\mathrm{Pt}(779)$, which has the same terrace structure of Pt(997) but steps consisting of $\{100\}$ instead of $\{111\}$ microfacets. ${ }^{37}$

If the He beam incidence angle is reduced slightly $\left(72^{\circ}\right.$ $<\theta_{i}<85^{\circ}$ ) the reflected He intensity has a contribution from both the terraces and the steps. ${ }^{36}$ For $\theta_{i}=83^{\circ}$ a broad maximum appears at around 0.5 ML (see Fig. 3). This maximum

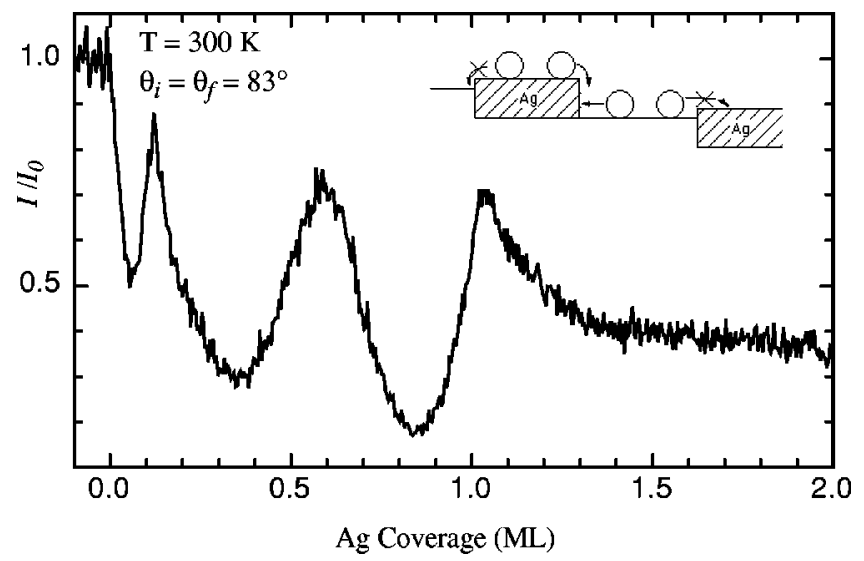

FIG. 3. TEAS intensity during Ag deposition at $300 \mathrm{~K}, \theta_{i}=\theta_{f}$ $=83^{\circ}, \lambda_{\mathrm{He}}=1.08 \AA$. The deposition rate is the same as in Fig. 2. A $4^{\circ}$ variation in the total scattering angle with respect to the lower curve in Fig. 2 considerably changes the reflectivity of the surface. The broad maximum at around 0.5-0.6 ML originates from a better periodicity of the surface due to the inhibited diffusion processes depicted in the inset (see text for further explanation). The peak at $1 \mathrm{ML}$ originates from the terrace contribution to the reflectivity, which is higher for Ag than for Pt.

reflects the evolution of the terrace width distribution during the growth of the first monolayer. Due to the adatom confinement effects described above the standard deviation $\sigma$ of the terrace width distribution reaches a minimum for $\theta_{\mathrm{Ag}}$ $=0.5 \mathrm{ML}$; this process is analogous to a step debunching effect where the velocity of each step is proportional to the width of the terrace it has in front. ${ }^{38}$ As a result, the better surface periodicity narrows the grazing incidence diffraction peak and increases its intensity. The 0.5 ML peak thus adds valuable information on the growth process, marking the presence of effective diffusion barriers across heterosteps.

In Fig. 3 there is a third maximum at $\theta_{\mathrm{Ag}}=1 \mathrm{ML}$. As discussed in Ref. 36 this maximum disappears at a more grazing incidence [see Fig. 2(b)]; its intensity comes from the Ag covered terrace, which has a higher reflectivity than bare $\mathrm{Pt}$.

By analyzing the TEAS curves recorded at different angles of incidence we have a means to detect the formation of monatomic wires and the quality of the surface periodic pattern. From this knowledge, we can prepare samples in the interesting range of temperature and coverage to study the respective atomic details with the STM.

\section{B. STM measurements}

STM images have been taken only for the $\mathrm{Ag} / \mathrm{Pt}(997)$ system. It is known from studies of $\mathrm{Ag} / \mathrm{Pt}(111)$ that $\mathrm{Ag}$ and Pt are chemically distinguishable by STM due to a positive height difference between the first Ag layer and a Pt step, which is largely independent from the tunneling conditions. ${ }^{39,40}$ In images like Fig. 1(a) however, it is virtually impossible to detect small amounts of adatoms at step edges. To analyze the initial stages of row growth we have to reduce our field-of-view to less than $200 \times 200 \AA^{2}$. Figure 4 is taken after deposition of $0.03 \mathrm{ML} \mathrm{Ag}$ at $340 \mathrm{~K}$. Two 1D $\mathrm{Ag}$ islands are visible attached to the lower step edge of two adjacent steps (see arrows). At $340 \mathrm{~K}$ the $\mathrm{Ag}$ adatoms im- 


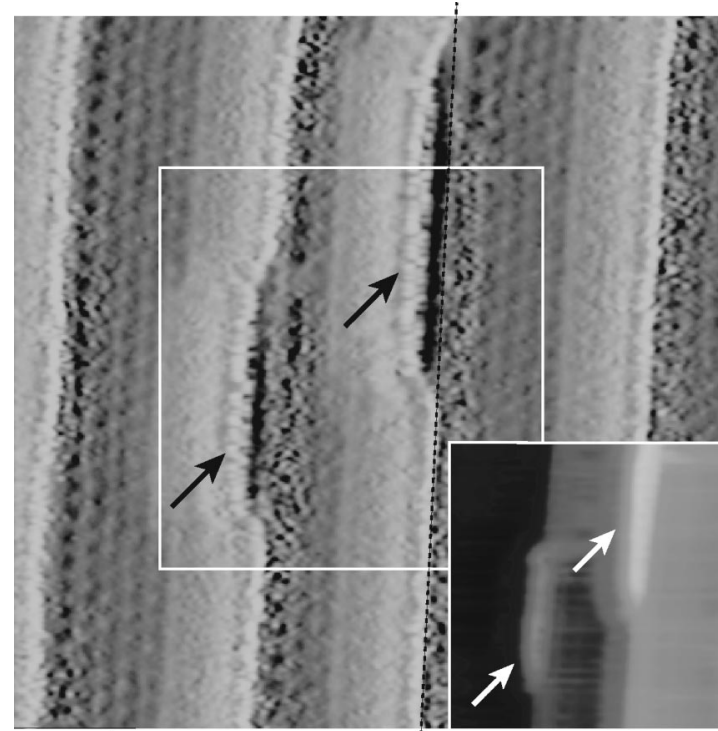

$10 \AA$

FIG. 4. $\partial z / \partial x$ image of the row formation process at $T$ $=340 \mathrm{~K}$. Step-down direction is from right to left, one of the Pt step edges is marked by a dotted line. Two Ag 1D aggregates (indicated by the arrows) are attached to the lower step edges. $\theta_{\mathrm{Ag}}$ $=0.03 \mathrm{ML}, F=3 \times 10^{-3} \mathrm{ML} / \mathrm{s}, I=4.6 \mathrm{nA}, V=6 \mathrm{mV}$. In the inset, a constant current image of the same spot shows the STM contrast between $\mathrm{Ag}$ and Pt.

pinging on the surface can easily migrate to step sites where they start diffusing along the Pt step edges. With increasing Ag coverage they will meet other Ag atoms and accommodate in stable 1D aggregates. The length of the stable 1D nucleus will generally depend on the sample temperature and on the deposition rate and it has not been investigated here. Eventually, these islands coalesce and form a continuous row along the Pt step edge as shown in Fig. 5. From images like Fig. 5 taken at $T \geqslant 300 \mathrm{~K}$ we note further that the first $\mathrm{Ag}$ row follows the Pt step contour in a perfect pseudomorphic way. This means that the Ag wire is a true 1D structure whose length is ultimately limited by the kink density of the substrate. Therefore, the sample miscut determines not only the average separation between the wires but also their longitudinal coherence.

\section{Ag wire growth vs $T$}

As Ag grows pseudomorphically on $\mathrm{Pt}(111)$ in a wide range of temperatures without intermixing with the substrate, $\mathrm{Ag} / \mathrm{Pt}(997)$ is regarded as a model system in our study of metal chain formation. Figure 6 shows the TEAS curves recorded at grazing incidence $\left(\theta_{i}=85^{\circ}\right)$ during submonolayer $\mathrm{Ag}$ deposition at different temperatures. As discussed in Sec. III A, the peak at $\sim 0.13 \mathrm{ML}$ corresponds to the completion of the first $\mathrm{Ag}$ row. The temperature evolution of this peak shows that monatomic row growth occurs for $150 \mathrm{~K} \leqslant T$ $\leqslant 550 \mathrm{~K}$. Although this result depends on the particular system under examination, it shows that step decoration is a valid method to obtain monatomic wires for a wide range of temperatures. However, even in the allowed temperature range, the "quality" of the monatomic wires can vary and the wire pattern for $\theta_{\mathrm{Ag}}>0.13 \mathrm{ML}$ changes as well. The
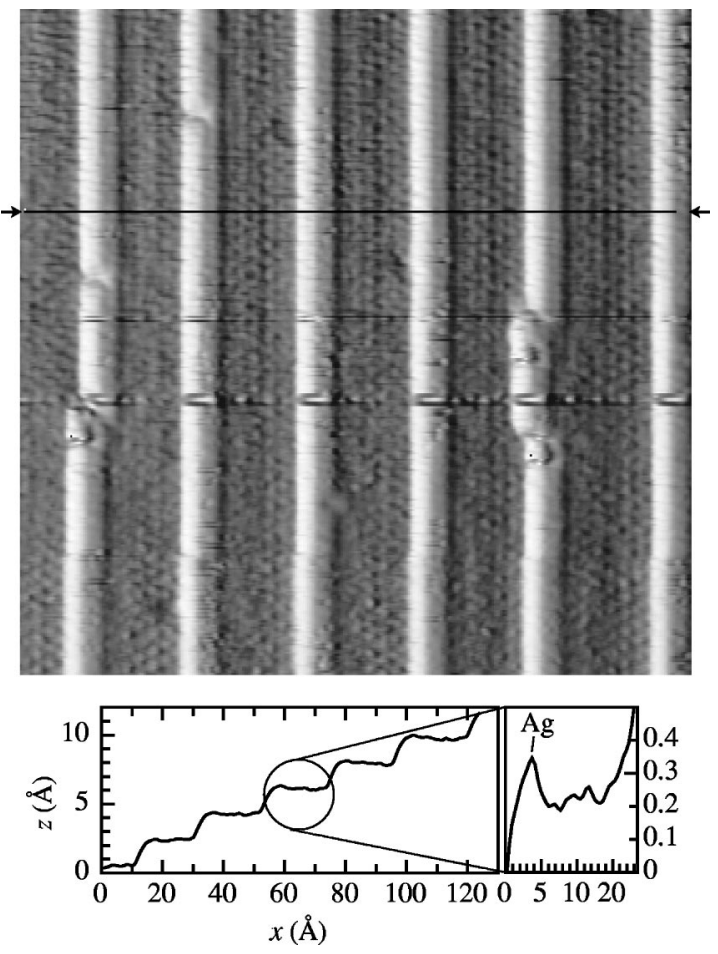

FIG. 5. $\partial z / \partial x$ image of single monatomic Ag wires decorating the Pt step edges. Ag is distinguishable from Pt because of its larger apparent height as shown in the constant current line scan at the bottom and by the luminosity contrast along the steps. $\theta_{\mathrm{Ag}}$ $=0.13 \mathrm{ML}, \quad F=3 \times 10^{-3} \mathrm{ML} / \mathrm{s}, \quad T=400 \mathrm{~K}, \quad I=2.85 \mathrm{nA}, \quad V$ $=5 \mathrm{mV}$.

dependence of row growth on the deposition rate $F$ has been investigated at $300 \mathrm{~K}: \mathrm{He}$ curves measured for 3 $\times 10^{-4} \mathrm{ML} / \mathrm{s} \leqslant F \leqslant 2 \times 10^{-2} \mathrm{ML} / \mathrm{s}$ do not show any sizable difference, although we cannot exclude a different low temperature behavior. In the following, Ag growth is analyzed starting from low temperature.

On Pt(111) single-Ag adatoms are mobile above $50 \mathrm{~K} ; \mathrm{Ag}$ dimers on the same surface are immobile and stable up to $110 \mathrm{~K}^{41}$ Therefore for $50 \mathrm{~K}<T<110 \mathrm{~K} \mathrm{Ag}$ adatoms can diffuse on the terrace until they meet a second adatom and form a stable dimer without attaching to a step. On the other hand, because the terrace width of $\mathrm{Pt}(997)$ is small compared to the mean free paths of $\mathrm{Ag}$ adatoms at $110 \mathrm{~K}(\approx 100 \AA$ as inferred by the mean island density measured by Brune $e t$ al. in Ref. 41), most adatoms can migrate to step sites even at lower temperatures. But step attachment is not the only necessary condition to form a 1D wire. At $T<150 \mathrm{~K} \mathrm{Ag}$ adatoms do not have enough thermal energy to accommodate into the minimum-energy configuration, which is the pseudomorphic decoration of a Pt step: they migrate to a step but stick to the first site of contact. Thus, the wire formation is kinetically hindered by slow edge- and corner-diffusion processes. Figure 7 shows such a situation where Ag islands grow attached to the step edges with an irregular contour following deposition at $120 \mathrm{~K}$.

We now proceed to examine row growth in the allowed temperature range: $150 \mathrm{~K} \leqslant T \leqslant 550 \mathrm{~K}$. As we demonstrated in Sec. III B, Figs. 4 and 5, row growth progresses via incorporation of adatoms in 1D stable nuclei attached to the step edges. Perfect row growth implies that all the Ag atoms mi- 


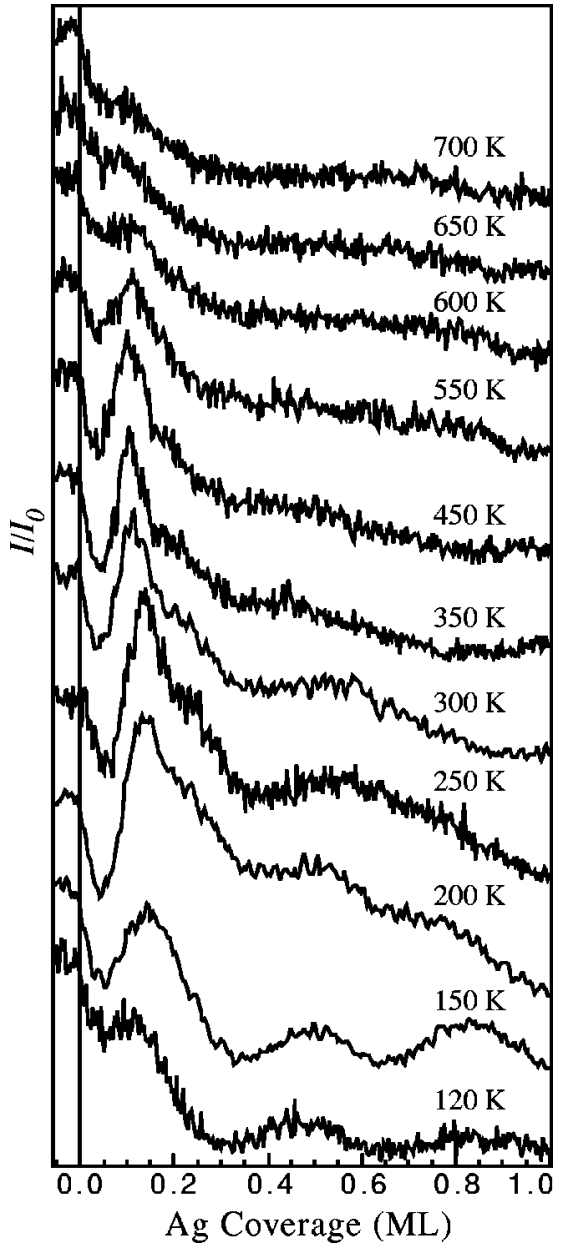

FIG. 6. TEAS intensity during deposition of $\mathrm{Ag}$ at different temperatures; $\theta_{i}=\theta_{f}=85^{\circ}, \lambda_{\mathrm{He}}=1.01 \AA, F=3 \times 10^{-3} \mathrm{ML} / \mathrm{s}$ for all curves. The sequence shows the temperature evolution of the peak at $\sim 0.13-0.17 \mathrm{ML}$ that corresponds to the formation of the first Ag row. The coverage shift of the peak maximum with respect to the nominal $0.13 \mathrm{ML}$ value of a monatomic wire is due to imperfect row growth at low temperature, see Fig. 8 and text.

grating to the steps are incorporated in the growing row. However, a plot of the coverage corresponding to the first peak maximum in Fig. 6 vs deposition temperature shows that this occurs only for $T>250 \mathrm{~K}$ as coincidence with the monatomic row nominal coverage of $0.13 \mathrm{ML}$ is attained (see Fig. 8). This implies that at $T \leqslant 250 \mathrm{~K}$ the $\mathrm{Ag}$-Ag edge and corner trapping diffusion barriers are still effective in preventing $\mathrm{Ag}$ adatoms that stick to a $\mathrm{Ag}$-covered step to migrate to the bare $\mathrm{Pt}$ step sites. The rate-limiting processes for wire smoothing are most likely kink breakup, corner breakup and corner crossing of $\mathrm{Ag}$ atoms attached to $\mathrm{Ag}$ decorated steps (indicated by $K, C_{b}$, and $C_{c}$, respectively in the inset in Fig. 8). Density-functional calculations for the $\mathrm{Al} / \mathrm{Al}(111)$ system have indeed shown kink and corner breakup as well as corner crossing to have the highest barriers among low-symmetry diffusion processes. ${ }^{42}$ The above can be considered to be relevant in the general case for wire formation as the same trend is observed for $\mathrm{Cu}$ although with a lower temperature threshold. A further way to rearrange 2D islands attached to the step edges into a perfect row is adatom 2D evaporation from the step to the terrace and subsequent recondensation. Evaporation of $\mathrm{Ag}$ atoms from 2D Ag

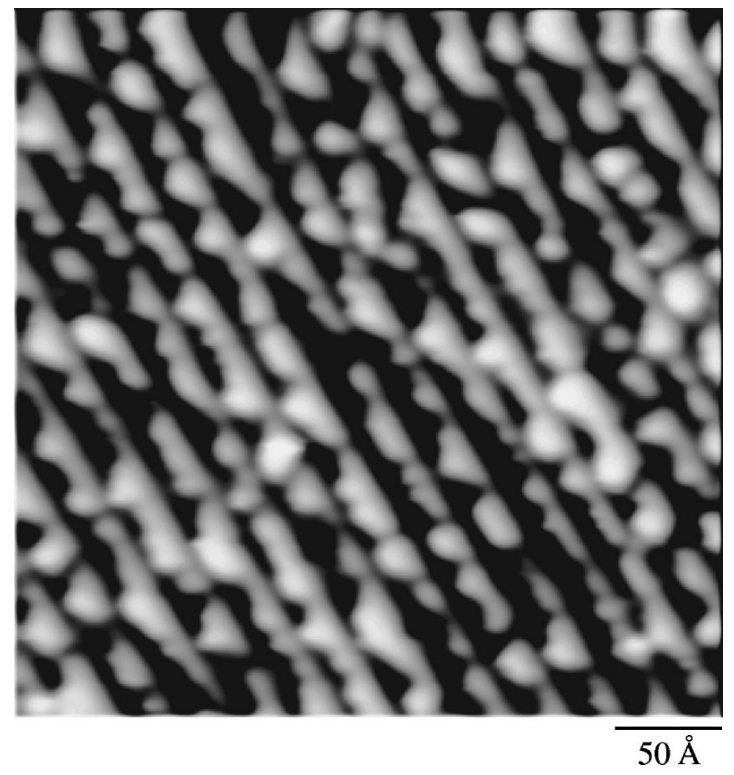

FIG. 7. STM constant current image taken after deposition of $0.5 \mathrm{ML}$ of $\mathrm{Ag}$ at $120 \mathrm{~K}, F=3 \times 10^{-3} \mathrm{ML} / \mathrm{s}, \quad I=1.1 \mathrm{nA}, V$ $=0.1 \mathrm{~V}$.

islands on $\mathrm{Pt}(111)$ has been shown to set in with temperatures exceeding $110 \mathrm{~K}$ (Ref. 14) and to lead to a dense 2D gas phase of $\mathrm{Ag}$ adatoms on large terraces at $T>300 \mathrm{~K}^{43}$ We conclude that the most regular Ag monatomic wires in terms of $1 \mathrm{D}$ character are grown above $250 \mathrm{~K}$, as shown in Fig. 5.

Increasing the temperature to obtain smooth wires has its limits. Although Ag has a higher surface free energy than Pt,

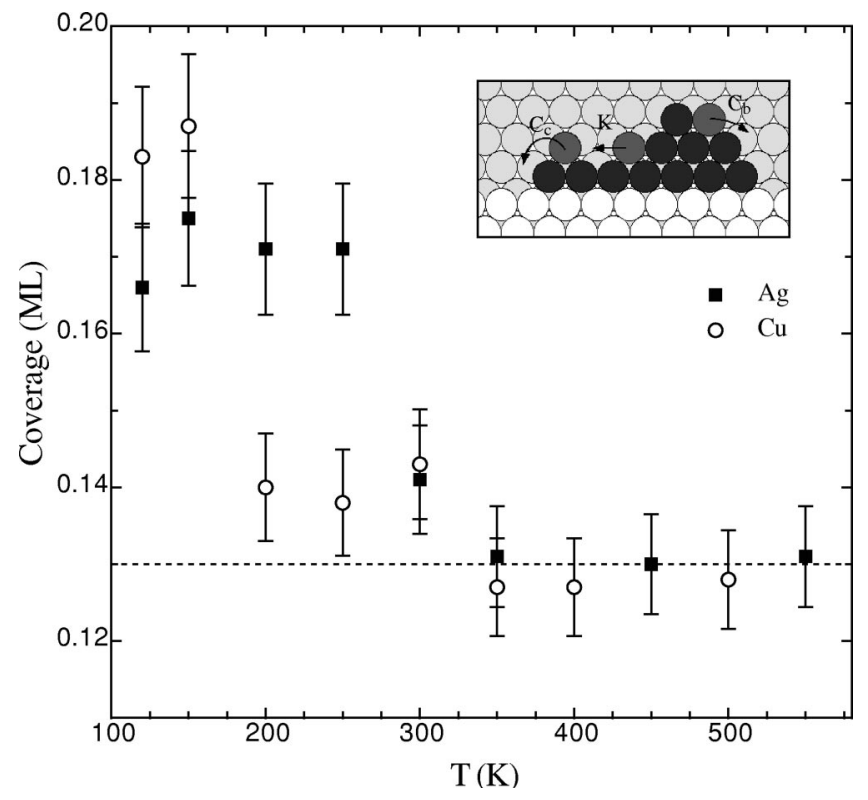

FIG. 8. Coverage corresponding to the first He reflectivity maximum in Fig. 6 for $\mathrm{Ag}$ and Fig. 14 for $\mathrm{Cu}$ vs deposition temperature. The coverage is calibrated with the procedure outlined in Sec. III A at $300 \mathrm{~K}$. The nominal coverage of a single monatomic row on $\mathrm{Pt}(997)$ is $0.13 \mathrm{ML}$ as indicated by the dotted line. Completion of the first row at coverages greater than $0.13 \mathrm{ML}$ indicates that the growing rows are not smooth because of slow kink, corner breaking, and corner crossing rates $\left(K, C_{b}\right.$, and $C_{c}$ in the inset). 


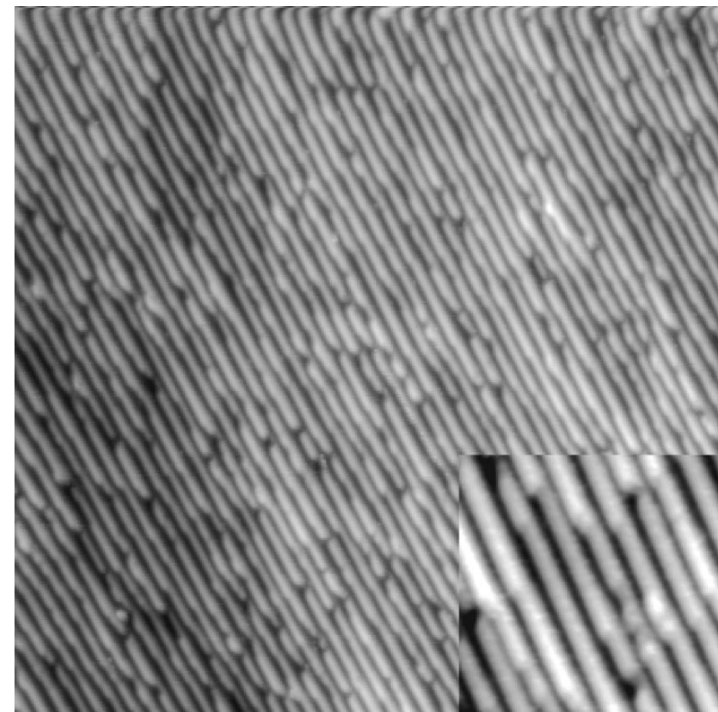

$100 \AA$

FIG. 9. Constant current image of $0.5 \mathrm{ML} \mathrm{Ag} \mathrm{deposited} \mathrm{at} T$ $=230 \mathrm{~K}, F=3 \times 10^{-3} \mathrm{ML} / \mathrm{s}, I=1.3 \mathrm{nA}, V=0.1 \mathrm{~V}$; the inset shows the Ag edges in more detail. A tip broadening effect enlarges the apparent width of the $\mathrm{Ag}$ stripes.

intermixing confined to the outer layer of the surface occurs for $T \geqslant 600 \mathrm{~K}^{39}$ As shown by Röder et al. in Ref. 39, diffusion of $\mathrm{Ag}$ into the $\mathrm{Pt}$ topmost layer proceeds via the step edges; roughening of the Ag-Pt interface at the step edges increases the defect density seen by the He atoms. Accordingly, the first row peak in Fig. 6 gradually decreases in intensity and finally disappears as the temperature raises to more than $600 \mathrm{~K}$. The upper limit for wire growth is evidently determined by the surface alloying temperature of the system. One must be careful, though, that intermixing at step edges might be effective before that alloying takes place homogeneously on the terraces. In Sec. III E we will see that it might also be desirable to stay far below the alloying temperature to maintain the wire pattern uniformity by avoiding diffusion of adatoms across different terraces.

\section{Ag wire growth vs coverage}

As the coverage increases to more than a single monatomic wire per terrace, Ag can either grow row-by-row or form rough stripes, imitating a Stranski-Krastanov growth mode in 1D. The latter growth mode has been observed, e.g., by Mo and Himpsel for $\mathrm{Cu}$ on a stepped W(110) surface. ${ }^{44}$ We find that, up to $0.5-0.7 \mathrm{ML}$, the Ag stripes, although not perfectly continuos, have smooth edges (see Fig. 9), but that for higher coverages the Ag edge becomes rough, as already mentioned in Sec. III A. The roughening of the Ag edge is evident from the comparison of Figs. 9 and 10 taken after deposition of 0.5 and $0.85 \mathrm{ML} \mathrm{Ag}$, respectively, at $230 \mathrm{~K}$. Incidentally, we point out the analogy between Ag growth on $\operatorname{Pt}(111)$ and on $\operatorname{Pt}(997)$ : on $\operatorname{Pt}(111)$ a transition from 2D layer-by-layer growth to 3D growth is observed above a critical coverage threshold; ${ }^{40}$ on $\mathrm{Pt}(997)$ we observe a transition from 1D row-by-row growth to rough 2D growth above $0.5 \mathrm{ML}$.

Roughening of the Ag edge can either be of kinetic or of thermodynamic origin. In the first case the process that limits

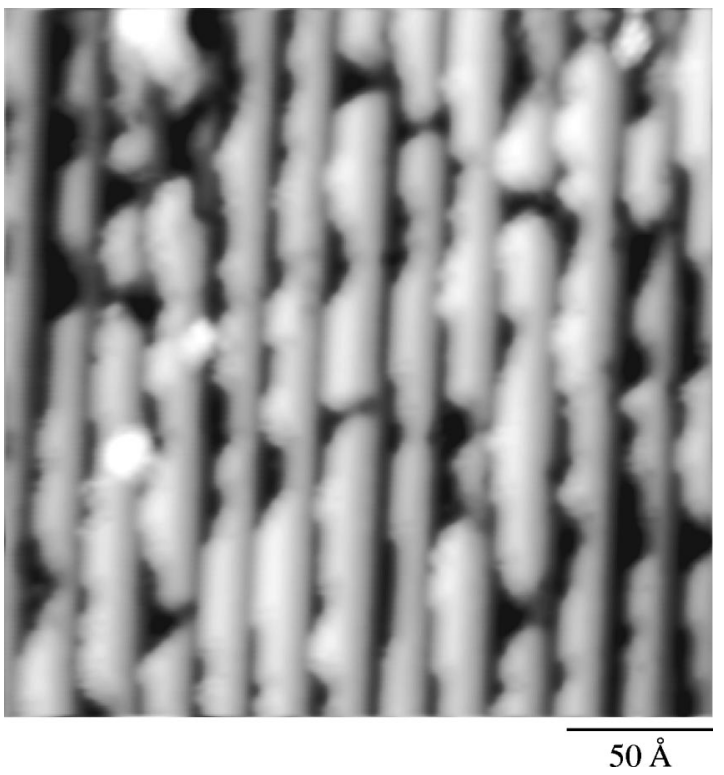

FIG. 10. Constant current image of $0.85 \mathrm{ML} \mathrm{Ag}$ deposited at $T=230 \mathrm{~K}, F=3 \times 10^{-3} \mathrm{ML} / \mathrm{s}, I=5.3 \mathrm{nA}, V=-7 \mathrm{mV}$; the $\mathrm{Ag}$ edges are rough compared to the inset in Fig. 9.

the reshaping of the rough $\mathrm{Ag}$ stripe into a smooth one might be the progressive closing of diffusion channels as the stripe edge approaches the lower terrace. Ag adatoms on the upper terrace are confronted to a strong repulsive barrier from the the Pt-Ag heterostep (see inset in Fig. 3). ${ }^{40}$ Such a repulsion can enhance diffusion barriers along the stripe edge and limit rearrangement by evaporation and recondensation. Moreover, the diffusion barriers along the Ag stripes and across the stripe corners might become larger due to an increasing outward relaxation of the stripe edge atoms caused by the strain accumulated as the Ag stripes grow wider. Since diffusion is generally slower along $\{111\}$ than along $\{100\}$ faceted steps ${ }^{42,45,46}$ we expect $\mathrm{Ag}$ stripes grown on $\mathrm{Pt}(779)$ to be smooth at higher coverages with respect to the stripes grown on Pt(997). Preliminary measurements indicate that this is indeed the case for $\mathrm{Ag} / \mathrm{Pt}(779)$, as mentioned in Sec. III A. ${ }^{37}$

Thermodynamics could also play a role in the observed roughening near monolayer completion. The minimization of the misfit strain energy between the Pt substrate and the Ag adlayer might lead to the formation of irregular structures where Ag atoms are less compressed compared to straight stripes. At present, we have no means to rule out kinetic vs thermodynamic arguments; the persistence of rough growth up to $430 \mathrm{~K}$ at least implies large rearrangement barriers if the state of the system is determined by kinetic limitations.

\section{E. Periodic patterns of Ag wires}

Besides the 1D character of wires grown by step decoration, their uniformity and their spatial distribution on the surface are other important issues. This is especially true if one wants to prepare samples for investigation by integral probes such as in, e.g., photoemission or photoabsorption spectroscopy experiments. It is clear that the average spacing (or density) of the wires is determined by the sample miscut and that the width of the spacing distribution is determined by the accuracy and the homogeneity of the sample miscut. The same is true for the wire thickness, however, only when 


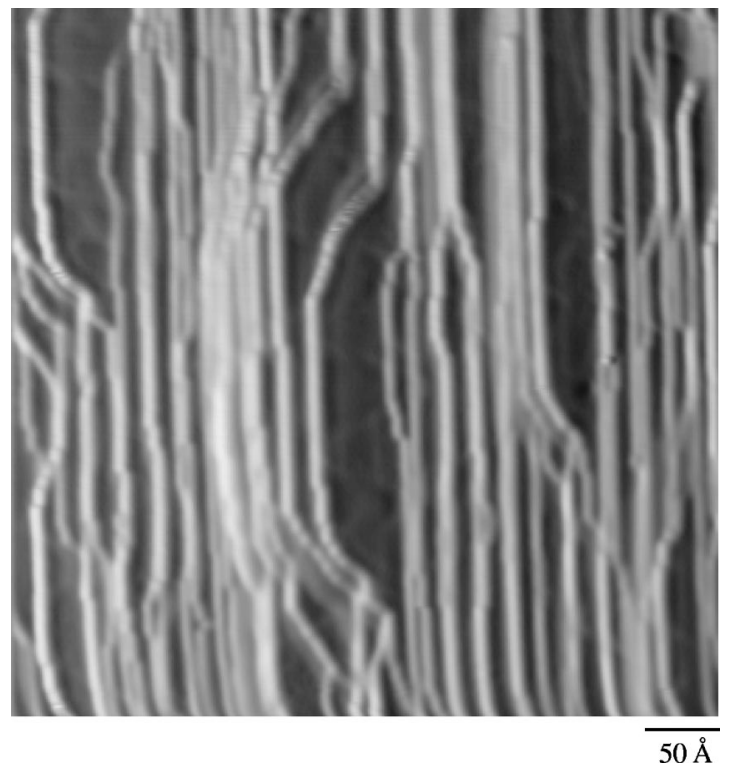

FIG. 11. $\partial z / \partial x$ image taken after deposition of $5 \mathrm{ML} \mathrm{Ag}$ at $T$ $=430 \mathrm{~K}$. Faceting results from Ag atoms crossing the Ag-Pt lateral interface at steps causing step bunching. Note that the Ag facets are not wider than $\sim 80 \AA . I=1 \mathrm{nA}, V=0.62 \mathrm{~V}$.

the adlayer coverage on each terrace is proportional to the terrace width, i.e., in the absence of interlayer mass transport. Assuming that our substrate is ideal, in order to grow uniform wires of the same (average) thickness we have to work at substrate temperatures that are below the threshold of heterostep crossing (referred to as interlayer diffusion in the following).

The temperature at which interlayer diffusion becomes significant is determined by TEAS. As discussed in Sec. III A, the TEAS measurements taken at $\theta_{i}=\theta_{f}=83^{\circ}$ show a peak at $\sim 0.5$ ML coverage that is due to terrace confinement of adatoms (see Fig. 3). The intensity of this peak in the Ag case decreases for $T>400 \mathrm{~K}$, indicating that Ag adatoms eventually acquire enough thermal energy to overcome the heterostep barriers. The analysis of the STM topograph shown in Fig. 11 confirms this conclusion. At coverages larger than $1 \mathrm{ML}, \mathrm{Ag}$ atoms diffusing across adjacent terraces can give rise to step bunching and faceting. Since the formation of (111) facets disrupts the periodicity of the surface, the diffraction pattern of the reflected He beam is also modified with respect to that of a vicinal (997) surface. Figure 12 shows the comparison between a spectrum taken after deposition of $20 \mathrm{ML} \mathrm{Ag}$ at $450 \mathrm{~K}$ (solid line) and one of the clean Pt(997) surface (dotted line). At non-grazing angles of incidence, specular reflection arises from the scattering of $\mathrm{He}$ atoms by flat (111) facets; the clean Pt(997) diffraction spectrum includes only the $n \neq 0$ diffraction orders, the signature of the periodic structure of the surface.

If we define the threshold temperature at which heterostep crossing becomes active as the temperature where crossing takes place once per second, we can estimate the diffusion barrier $E_{h}$ for this process as

$$
E_{h}=\ln \left(\nu_{0}\right) k_{B} T,
$$

where $k_{B}$ is the Boltzmann constant and $\nu_{0}$ is the usual prefactor. By taking $\nu_{0}=6 \times 10^{12}$ as in Ref. 42 , and $T$

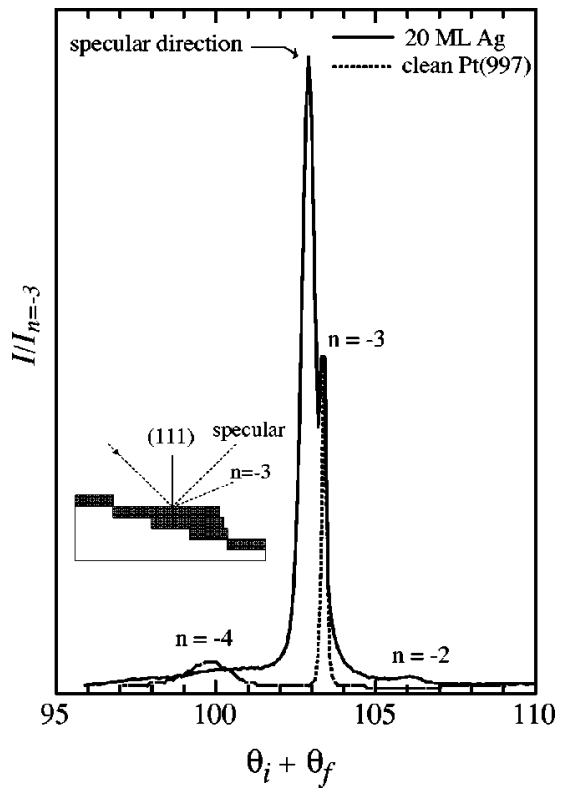

FIG. 12. Diffraction pattern of $20 \mathrm{ML} \mathrm{Ag} \mathrm{deposited} \mathrm{on} \mathrm{Pt}(997)$ at $T=450 \mathrm{~K}$ (solid line) compared to that of the bare $\mathrm{Pt}(997)$ surface (dotted line). The intensity in the specular direction is due to faceting of the Ag adlayers. Both curves are arbitrarily normalized to the $n=-3$ peak. During the scan $\theta_{i}=58.0^{\circ}$ is kept fixed, while $\theta_{f}$ varies; $\lambda_{\mathrm{He}}=1.01 \AA$.

$=400 \mathrm{~K}$ we calculate $E_{h}$ to be $0.9 \mathrm{eV}$ for diffusion of $\mathrm{Ag}$ atoms across Pt-Ag step boundaries. We note that even higher temperatures for crossing the Pt-Ag border are required on $\mathrm{Pt}(111)$, where the large barrier for heterostep crossing has been attributed to the binding energy difference of $\mathrm{Ag} / \mathrm{Pt}(111)$ with respect to $\mathrm{Ag} / 1 \mathrm{ML} \mathrm{Ag} / \mathrm{Pt}(111)$ and to compressive strain in the Ag layer. ${ }^{40}$

From the results presented in this section we conclude that the optimal temperature range for patterning $\mathrm{Pt}(997)$ with Ag nanowire arrays is between 250 and $400 \mathrm{~K}$.

\section{F. Cu Wire Growth}

$\mathrm{Cu}$ growth on $\mathrm{Pt}(997)$ is first compared to the flat (111) surface and subsequently analyzed in terms of the wire growth. The growth of $\mathrm{Cu}$ on $\mathrm{Pt}$ vicinal substrates appears to be quite different with respect to the (111) surface. Holst et al. reported in a TEAS-STM study of $\mathrm{Cu}$ on $\mathrm{Pt}(111)$ (Ref. 47) no oscillations in the He scattering reflectivity during growth at $340 \mathrm{~K}$. They attributed the oscillation damping to incomplete coalescence of the first layer $\mathrm{Cu}$ islands and to the formation of a dislocation network at $\theta_{\mathrm{Cu}}=2 \mathrm{ML}$. On $\mathrm{Pt}(997)$, in contrast, we found oscillations in the deposition curves at least up to $4 \mathrm{ML}$ in the same temperature range, with damping occurring only for $\theta_{\mathrm{Cu}} \geqslant 2$ ML.

Figure 13 shows the $\mathrm{He}$ intensity during $\mathrm{Cu}$ deposition at $350 \mathrm{~K}$ : the first maximum corresponds to the formation of the first $\mathrm{Cu}$ row at $0.13 \mathrm{ML}$. This is confirmed by the grazing incidence curves in Fig. 14, which show the first row peak as a function of growth temperature. The reason why the first row formation gives rise to an intensity maximum in the non-grazing geometry, in contrast to the $\mathrm{Ag}$ case, is probably due to a bending of the He-surface scattering potential near the step edges induced by $\mathrm{Cu}$ atoms. The second maximum 


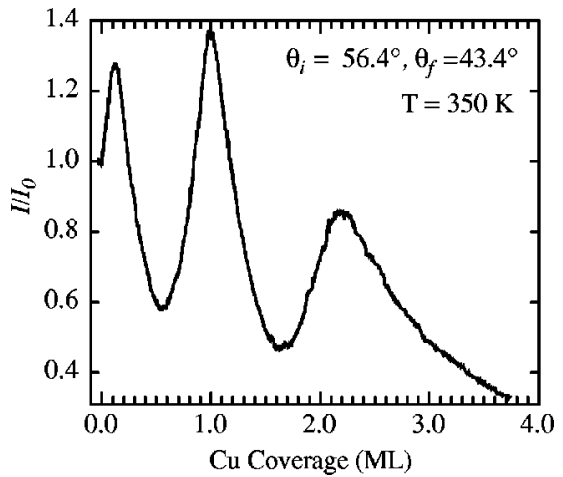

FIG. 13. Normalized intensity of the reflected He beam during deposition of $\mathrm{Cu}$. The second peak reflects the completion of the first $\mathrm{Cu}$ adlayer and is used to calibrate the deposition rate for the curves reported in Fig. 14. $\lambda_{\mathrm{He}}=1.01 \AA, F=1.7 \times 10^{-3} \mathrm{ML} / \mathrm{s}$.

in Fig. 13 corresponds to the monolayer completion and serves as a coverage calibration for the deposition curves. Its intensity is larger than the bare Pt signal $\left(I_{0}\right)$ because of the higher Debye temperature of $\mathrm{Cu}$. Damping occurs only from the second layer, indicating that the first $\mathrm{Cu}$ layer is complete and pseudomorphic with respect to the substrate. The better layer-by-layer growth mode on $\operatorname{Pt}(997)$ with respect to the (111) surface is related to its very short terrace width that renders second layer nucleation less likely. Also the formation of the $(13 \times 13)$ fcc-hcp dislocation network observed for $\theta_{\mathrm{Cu}}=2 \mathrm{ML}$ on $\mathrm{Pt}(111)$ (Refs. 16 and 47) is presumably suppressed on $\mathrm{Pt}(997)$, explaining the persistance of the oscillations in the He curves for $\theta_{\mathrm{Cu}}>1 \mathrm{ML}$.

From the analysis of the grazing incidence curves taken during $\mathrm{Cu}$ deposition we see that row growth occurs down to $T=120 \mathrm{~K}$ and presumably even at lower temperatures. However, at $T=120 \mathrm{~K}$ the first row peak in Fig. 14 is broad and centered around $0.18 \mathrm{ML}$, indicating slow edge diffusion. In the case of $\mathrm{Cu}$, the transition to smooth row growth happens between $150 \mathrm{~K}$ and $200 \mathrm{~K}$ (see Fig. 8), i.e., at lower temperature than for $\mathrm{Ag}$. On the other hand, $\mathrm{Cu}$ row growth seems to degrade earlier with increasing temperature than in the Ag case. The intensity of the first row peak normalized to the bare Pt signal is already considerably diminished at 350 $\mathrm{K}$ with respect to the low temperature value, while it is constant up to $500 \mathrm{~K}$ in the $\mathrm{Ag}$ case. The reduced row reflectivity is attributed to $\mathrm{Cu}-\mathrm{Pt}$ mixing at the step interface. Dynamic work function measurements indicate the onset of intermixing between $\mathrm{Cu}$ and $\mathrm{Pt}(111)$ at $500 \mathrm{~K} .{ }^{48}$ Since intermixing is favored at step sites, it is likely to set in earlier on $\mathrm{Pt}(997)$. At $T=600 \mathrm{~K}$ the row peak disappears completely; $\mathrm{Cu}$ deposition results in alloyed structures, in agreement with experiments on $\mathrm{Cu} / \mathrm{Pt}(111) .^{48}$

For $\theta_{i}=83^{\circ}$ (Fig. 14) the $0.5 \mathrm{ML}$ peak is clearly visible below $200 \mathrm{~K}$. As in Sec. III E, we attribute the suppression of this peak at $200 \mathrm{~K}$ to the onset of heterostep crossing by $\mathrm{Cu}$ atoms. Therefore, $\mathrm{Cu}$ seems to be more mobile on $\mathrm{Pt}$ than $\mathrm{Ag}$, in terms of both edge and interlayer diffusion. Assuming the same prefactor as in the Ag case and taking $T=200 \mathrm{~K}$ in Eq. (1), the diffusion barrier across the Pt-Cu boundary at the steps is estimated to be $\sim 0.5 \mathrm{eV}$.

\section{CONCLUSIONS}

To summarize, we have shown that we can routinely grow high-density arrays of parallel nanowires with real-time con-

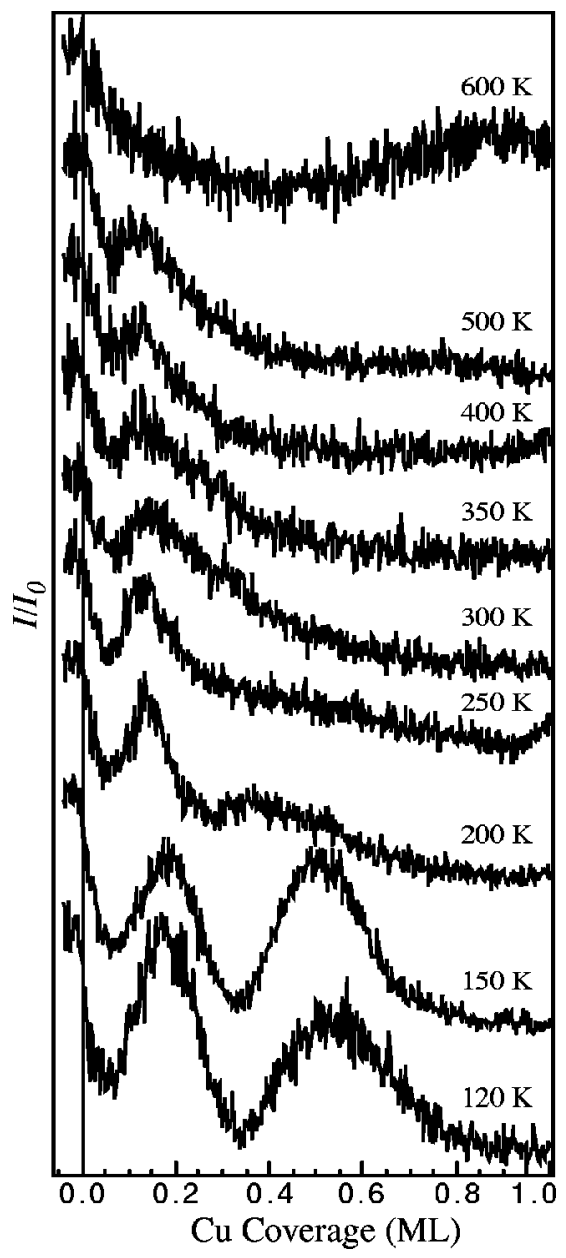

FIG. 14. TEAS intensity during deposition of $\mathrm{Cu}$ at different temperatures; $\theta_{i}=\theta_{f}=83^{\circ}, \lambda_{\mathrm{He}}=1.01 \AA, F=1.7 \times 10^{-3} \mathrm{ML} / \mathrm{s}$. The first peak at $\sim 0.13-0.18 \mathrm{ML}$ (first $\mathrm{Cu}$ monatomic row) is visible up to $T=500 \mathrm{~K}$. Note that the half monolayer peak (see Sec. III A for explanation) disappears at $T=200 \mathrm{~K}$ indicating the onset of $\mathrm{Cu}$ interlayer diffusion.

trol over monatomic wire deposition. The best temperature range to grow regular nanowire arrays is found for $T$ sufficiently high to ensure smooth wire formation and $T$ sufficiently low to avoid interlayer diffusion by the adatoms which would destroy the proportionality between terrace width and wire thickness. The optimal temperature range for $\mathrm{Ag}$ and $\mathrm{Cu}$ wire growth is situated between 250 and $400 \mathrm{~K}$, and between 150 and $200 \mathrm{~K}$, respectively.

The combination of TEAS and STM allows (1) to find the experimental parameters that lead to the best wire patterns in terms of 1D character, uniformity, spatial distribution and chemical identity, (2) to investigate diffusion processes that are relevant to step decoration and metal growth on stepped surfaces in general.

\section{ACKNOWLEDGMENTS}

P. Gambardella thanks O. Jeandupeaux and L. Bürgi for help with the STM setup and acknowledges financial support from the University of Genova, Italy. We thank J.V. Barth for careful reading of the manuscript. 
*Electronic address: pietro.gambardella@epfl.ch

†Also at Max-Planck-Institut für Festkörperforschung, Heisenbergstr. 1, D-70569 Stuttgart, Germany.

${ }^{1}$ M.F. Crommie, C.P. Lutz, and D.M. Eigler, Nature (London) 363, 524 (1993).

${ }^{2}$ Y. Hasegewa and P. Avouris, Phys. Rev. Lett. 71, 1071 (1993).

${ }^{3}$ F.J. Himpsel and J.E. Ortega, Phys. Rev. B 50, 4992 (1994).

${ }^{4}$ A. Biedermann, O. Genser, W. Hebenstreit, M. Shmid, J. Redinger, R. Podloucky, and P. Varga, Phys. Rev. Lett. 76, 4179 (1996).

${ }^{5}$ J. Li, W.-D. Schneider, and R. Berndt, Phys. Rev. B 56, 7656 (1997).

${ }^{6}$ L. Bürgi, O. Jeandupeux, A. Hirstein, H. Brune, and K. Kern, Phys. Rev. Lett. 81, 5370 (1998).

${ }^{7}$ B.J. Van Wees, H. van Houten, C.W.J. Beenakker, J.G. Williamson, L.P. Kouvenhoven, D. van der Marel, and C.T. Foxon, Phys. Rev. Lett. 60, 848 (1988).

${ }^{8}$ P. Grünberg, R. Schreiber, Y. Pang, M.B. Brodsky, and H. Sowers, Phys. Rev. Lett. 57, 2442 (1986).

${ }^{9}$ C. Carbone and S.F. Alvarado, Phys. Rev. B 36, 2433 (1987).

${ }^{10}$ M.N. Baibich, J.M. Broto, A. Fert, F. Nguyen Van Dau, F. Petroff, P. Eitenne, G. Creuzet, A. Friederich, and J. Chazelas, Phys. Rev. Lett. 61, 2472 (1988).

${ }^{11}$ G. Binasch, P. Grünberg, F. Saurenbach, and W. Zinn, Phys. Rev. B 39, 4828 (1989).

${ }^{12}$ S.S.P. Parkin, N. More, and K.P. Roche, Phys. Rev. Lett. 64, 2304 (1990).

${ }^{13}$ F.J. Himpsel, J.E. Ortega, G.J. Mankey, and R.F. Willis, Adv. Phys. 47, 511 (1998), and references therein.

${ }^{14}$ H. Röder, E. Hahn, H. Brune, J.P. Bucher, and K. Kern, Nature (London) 366, 141 (1993).

${ }^{15}$ V.E. Marsico, M. Blanc, K. Kuhnke, and K. Kern, Phys. Rev. Lett. 78, 94 (1997).

${ }^{16}$ H. Brune, M. Giovannini, K. Bromann, and K. Kern, Nature (London) 394, 451 (1998).

${ }^{17}$ G.A. Bassett, Philos. Mag. 3, 1042 (1958).

${ }^{18}$ H. Bethge, Surf. Sci. 3, 33 (1964).

${ }^{19}$ P.M. Petroff, A.C. Gossard, and W. Wiegmann, Appl. Phys. Lett. 45, 620 (1984).

${ }^{20}$ M. Tsuchiya, P.M. Petroff, and L.A. Coldren, Appl. Phys. Lett. 54, 1690 (1989).

${ }^{21}$ R. Nötzel, N.N. Ledentsov, L. Däweritz, K. Ploog, and M. Hohenstein, Phys. Rev. B 45, 3507 (1991).

${ }^{22}$ M. Sundaram, S.A. Chalmers, P.F. Hopkins, and A.C. Gossard, Science 254, 1326 (1991).

${ }^{23}$ M.S. Miller, H. Weman, C.E. Pryor, M. Krishnamurty, P.M. Petroff, H. Kroemer, and J.L. Merz, Phys. Rev. Lett. 68, 3464 (1992).

${ }^{24}$ M. Mundschau, E. Bauer, and W. Swiech, J. Appl. Phys. 65, 581 (1989).

${ }^{25}$ T. Jung, Y.W. Mo, and F.J. Himpsel, Phys. Rev. Lett. 74, 1641 (1994)
${ }^{26}$ T. Jung, R. Schlitter, J.K. Gimzewski, and F.J. Himpsel, Appl. Phys. A: Mater. Sci. Process. 61, 467 (1995).

${ }^{27}$ D.Y. Petrovykh, F.J. Himpsel, and T. Jung, Surf. Sci. 407, 189 (1998).

${ }^{28}$ J. de la Figuera, M.A. Huerta-Garnica, J.E. Prieto, C. Ocal, and R. Miranda, Appl. Phys. Lett. 66, 1006 (1995).

${ }^{29}$ J. Camarero, J. de la Figuera, L. Spendeler, X. Torrellas, J. Alvarez, S. Ferrer, J. J. de Miguel, J. M. García, O. Sánchez, J. E. Ortega, A. L. Vázquez de Parga, and R. Miranda, Magnetic Ultrathin Films, Multilayers and Surfaces, edited by A. Fert, H. Fujimori, E. Guntheralt, B. Heinrich, W. F. Egelhoff, Jr., E. E. Marinero, and R. L. White, MRS Symposia Proceeding No. 384 (Materials Research Society, Pittsburgh, 1995), p. 49.

${ }^{30}$ S. Papadia, M.C. Desjonqures, and D. Spanjaard, Phys. Rev. B 53, 4083 (1996); E.D. Williams and N.C. Bartelt, Science 251, 393 (1991)

${ }^{31}$ E. Hahn, H. Schief, V. Marsico, A. Fricke, and K. Kern, Phys. Rev. Lett. 72, 3378 (1994).

${ }^{32}$ K. Kuhnke, E. Hahn, R. David, P. Zeppenfeld, and K. Kern, Surf. Sci. 272, 118 (1992).

${ }^{33}$ H. Schief, Ph.D. thesis, Ecole Polytechnique Fédérale de Lausanne, 1995.

${ }^{34}$ O. Jeandupeux, L. Bürgi, A. Hirstein, H. Brune, and K. Kern, Phys. Rev. B 59, 15926 (1999).

${ }^{35}$ See, e.g., B. Poelsema, A.F. Becker, G. Rosenfeld, R. Kunkel, N. Nagel, L.K. Verheij, and G. Comsa, Surf. Sci. 272, 269 (1992), and references therein.

${ }^{36}$ M. Blanc, K. Kuhnke, V. Marsico, and K. Kern, Surf. Sci. 414, L964 (1998).

${ }^{37}$ P. Gambardella, M. Blanc, K. Kuhnke, and K. Kern (unpublished).

${ }^{38}$ D. Kandel and J.D. Weeks, Phys. Rev. Lett. 74, 3632 (1995).

${ }^{39}$ H. Röder, R. Schuster, H. Brune, and K. Kern, Phys. Rev. Lett. 71, 2086 (1993).

${ }^{40}$ H. Röder, K. Bromann, H. Brune, and K. Kern, Surf. Sci. 376, 13 (1997).

${ }^{41}$ H. Brune, H. Röder, C. Boragno, and K. Kern, Phys. Rev. Lett. 73, 1955 (1994).

${ }^{42}$ A. Bogicevic, J. Strömquist, and B.I. Lundqvist, Phys. Rev. Lett. 81, 637 (1998)

${ }^{43}$ H. Röder, H. Brune, J.P. Bucher, and K. Kern, Surf. Sci. 298, 121 (1993).

${ }^{44}$ Y.W. Mo and F.J. Himpsel, Phys. Rev. B 50, 7868 (1994).

${ }^{45}$ H. Brune, H. Röder, K. Bromann, K. Kern, J. Jacobsen, P. Stoltze, K. Jacobsen, and J. Nørskov, Surf. Sci. 349, L115 (1996).

${ }^{46}$ R. Stumpf and M. Scheffler, Phys. Rev. Lett. 72, 254 (1994).

${ }^{47}$ B. Holst, M. Nohlen, K. Wandelt, and W. Allison, Surf. Sci. 377-379, 891 (1997).

${ }^{48}$ B. Schaefer, M. Nohlen, and K. Wandelt, Surf. Sci. (to be published). 